ISSN 1420-3049

www.mdpi.com/journal/molecules

Article

\title{
Reactions of Some New Thienothiophene Derivatives
}

\section{Yahia Nasser Mabkhot ${ }^{1, *}$, Abdullah Mohammad Al-Majid ${ }^{1}$, Abdullah Saleh Alamary ${ }^{1}$, Ismail Warad $^{1}$ and Yamin Sedigi ${ }^{2}$}

1 Department of Chemistry, Faculty of Science, King Saud University, P.O. Box 2455, Riyadh 11451, Saudi Arabia

2 Department of Chemistry, Faculty of Science, Taibah University, P.O. Box 344, Medina, Saudi Arabia

* Author to whom correspondence should be addressed; E-Mail: yahia@ksu.edu.sa.

Received: 30 May 2011; in revised form: 15 June 2011 / Accepted: 15 June 2011 /

Published: 21 June 2011

\begin{abstract}
Facile and convenient syntheses of bisdimethylthieno[2,3-b]thiophen-2,5-diyl bis(oxazole-2-amine), bis( $1 H$-imidazol-2-amine), bis((3a)- $H$-indole),[1,2-a]pyrimidine), bis(1H-imidazo[1,2-b][1,2,4]triazole) and $\quad$ bis(9H-benzo[d]imidazo[1,2-a]imidazole $)$ derivatives incorporating a thieno[2,3-b]thiophene moiety from the versatile and readily accessible 1,1'(3,4-dimethylthieno[2,3-b]thiophene-2,5-diyl)-bis(2-bromo-ethanone) are described.
\end{abstract}

Keywords: bis(2-bromoethanone); bis-thieno[2,3-b]thiophene; bis(oxazole-2-amine); bis-heterocycles; imidazotriazole

\section{Introduction}

We have been interested for some time in the chemical and biological properties of thienothiophene derivatives [1-3]. Thienothiophenes have been developed for different purposes in the pharmaceutical field and have been tested as potential antitumor, antiviral, antibiotic and antiglaucoma drugs or as inhibitors of platelet aggregation [3-8]. In addition, thienothiophenes find potential applications in a wide variety of optical and electronic systems [9-11]. Recently, some conjugated thienothiophenes, structurally related to several current applications have been reported [12-19]. In continuation of these findings, we report herein the synthesis of some novel bis-heterocycles containing a 
thieno[2,3-b]thiophene moiety as a base unit and which are of interest as potential biologically active compounds or pharmaceuticals.

\section{Results and Discussion}

The synthetic procedures adopted to obtain the target compounds are outlined in Schemes 1, 2 and 3. Treatment of bis-2-bromoacetylthieno[2,3-b]thiophene derivative 1 [3] with urea, thiourea or guanidine in refluxing EtOH/TEA gave the novel bisthieno[2,3-b]thiophene derivatives 2a-c, respectively (Scheme 1). The structures of the products were deduced from their elemental analysis and spectral data. For example, the ${ }^{1} \mathrm{H}-\mathrm{NMR}$ spectrum of compound 2a revealed a singlet at $\delta 7.19$ characteristic of an oxazole $\mathrm{CH}$ proton. The IR spectra of 2a-c showed, in each case, the absence of the carbonyl bands found in $\mathbf{1}$ and the presence of new bands in the $3422-3385 \mathrm{~cm}^{-1}$ region due to $\mathrm{NH}_{2}$ and NH groups.

Scheme 1. Synthesis of bis-amino heterocycles derivatives 2a-b.
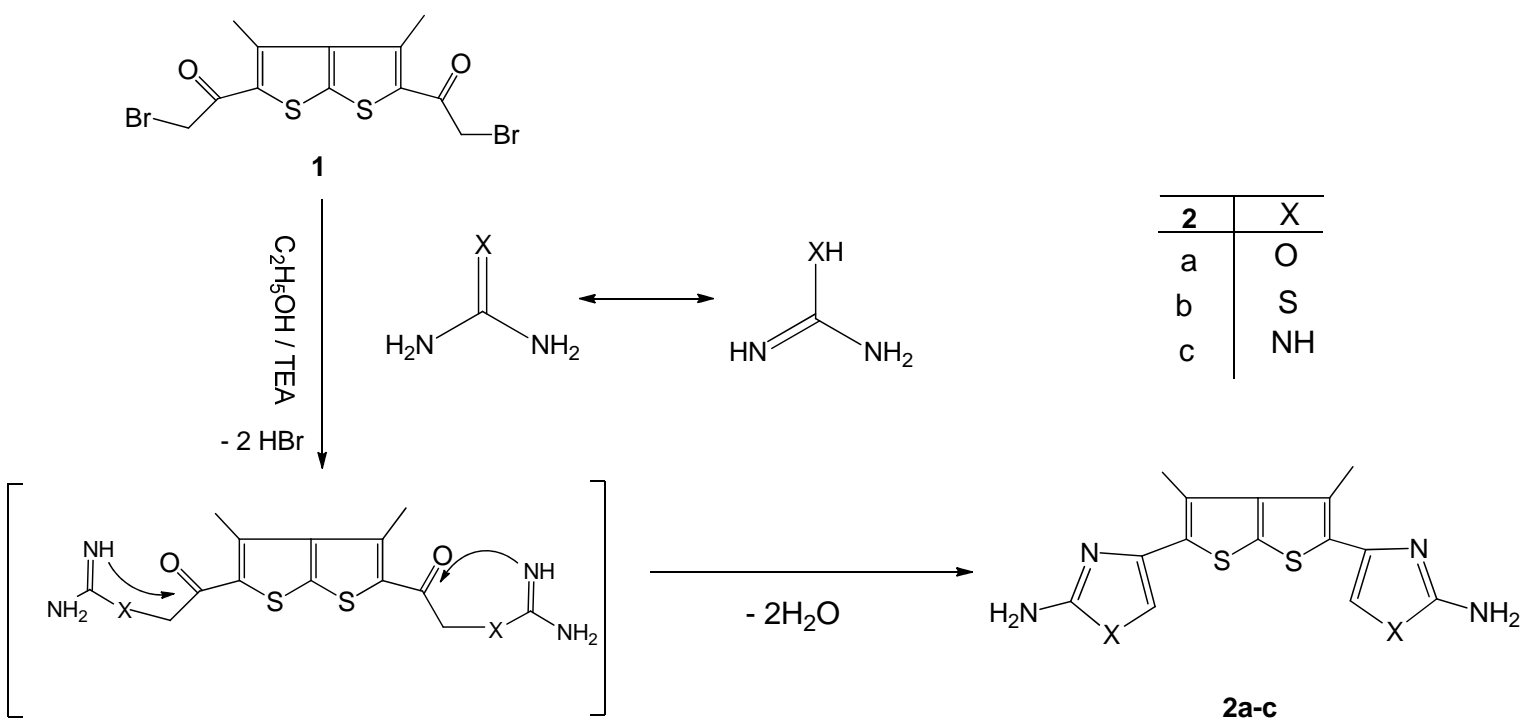

Treatment of compound $\mathbf{1}$ with aniline or with 2-aminopyrimidine in refluxing EtOH/TEA led to the novel bis-thieno[2,3-b]thiophene derivatives $\mathbf{4}$ and 5, respectively (Scheme 2), whose structures were confirmed on the basis of their elemental analyses and spectral data. The ${ }^{1} \mathrm{H}-\mathrm{NMR}$ spectrum of compound 5, for example, revealed signals at $\delta$ 7.43-7.80, characteristic of imidazole and pyrimidine $\mathrm{CH}$ protons. The IR spectrum of 5 lacked a carbonyl absorption band and the ${ }^{13} \mathrm{C}-\mathrm{NMR}$ spectrum revealed eleven types of carbon atoms (i.e., those of half the bisheterocycle), The IR spectrum of compound 4 showed a carbonyl absorption band at $1690 \mathrm{~cm}^{-1}$ [20]. Treatment of compound 1 with 4-amino-1,2,4-triazole in refluxing ethanol afforded 5,5'-(3,4-dimethylthieno[2,3-b]thiophene-2,5diyl)bis(1H-imidazo[1,2-b][1,2,4]triazole) (6, Scheme 3). The ${ }^{1} \mathrm{H}-\mathrm{NMR}$ spectrum of compound 6 displayed singlets at $\delta 2.22\left(\mathrm{CH}_{3}\right), \delta 7.80(2 \mathrm{H}, \mathrm{CH}$, imidazole $), 9.8(\mathrm{~s}, 2 \mathrm{C}, \mathrm{CH}$, triazole $)$ and $12.4(2 \mathrm{H}$, $\mathrm{NH}$, triazole). The ${ }^{13} \mathrm{C}-\mathrm{NMR}$ spectrum revealed nine types of carbon. The mass spectrum revealed a molecular ion peak at $\mathrm{m} / \mathrm{z} 380$, corresponding to $\mathrm{C}_{16} \mathrm{H}_{20} \mathrm{~N}_{8} \mathrm{~S}_{2}$. In a similar manner, when $\mathbf{1}$ was treated with 2-aminobenzimidazole, the corresponding compound 7 was obtained in high yield. 
Scheme 2. Synthesis of bis- thieno-thiophenes derivatives 4 and 5.

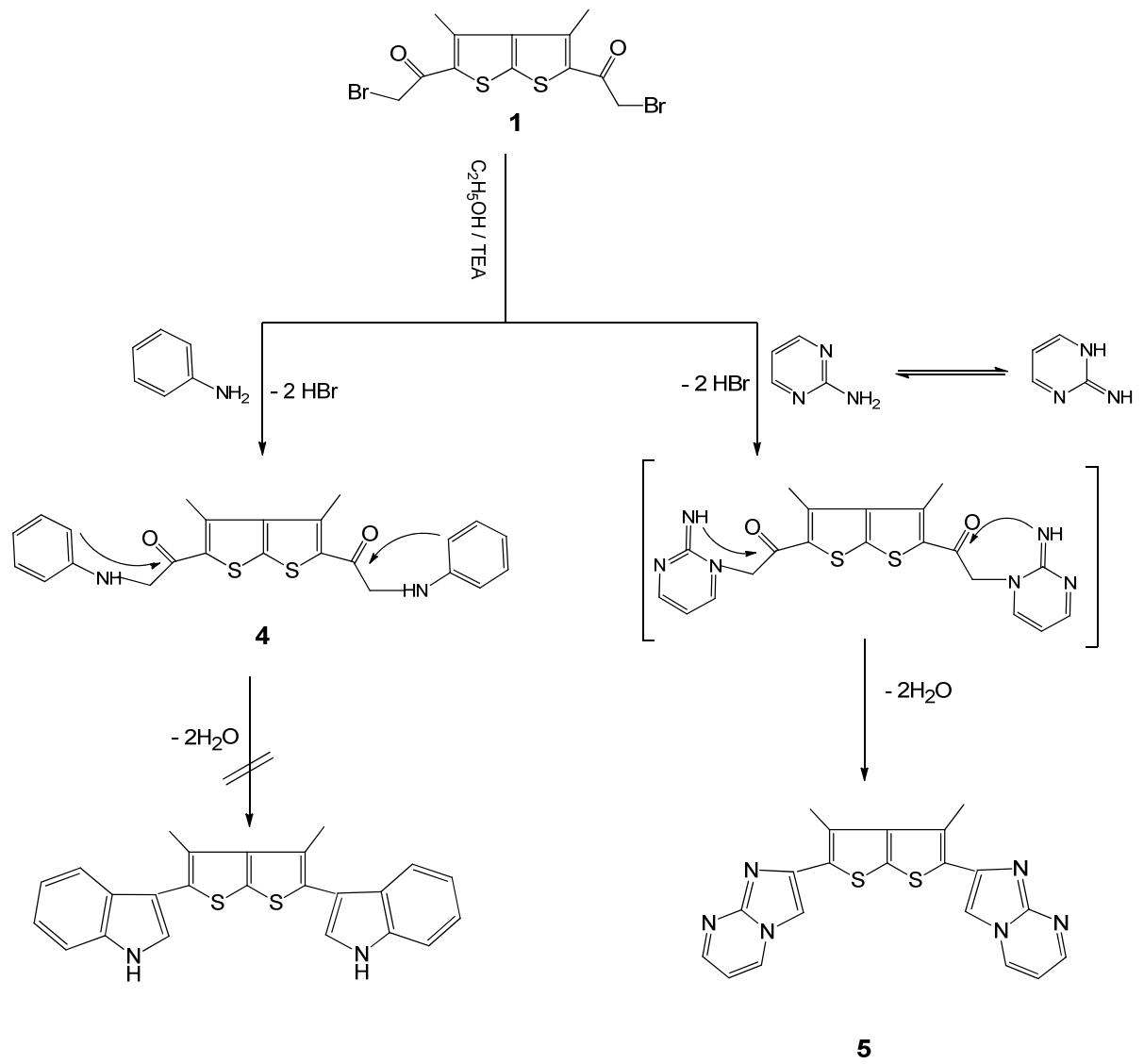

Scheme 3. Synthesis of bis- imidazole derivatives 6 and 7.
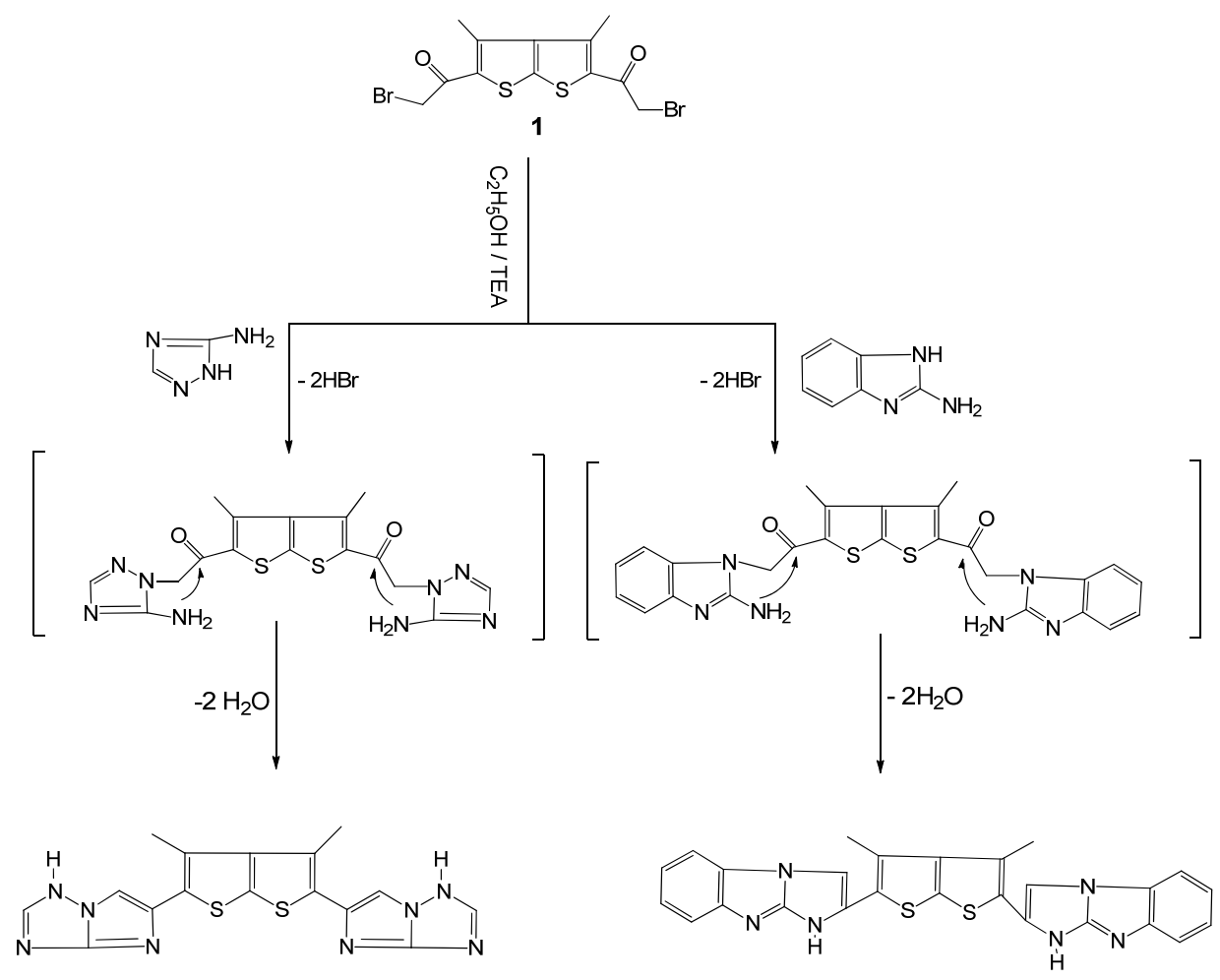


\section{Experimental}

\subsection{General}

All melting points were measured on a Koffler block melting point apparatus. IR spectra were measured as KBr pellets on a Perkin Elmer FT 1000 spectrophotometer. The NMR spectra were recorded in DMSO-d $\mathrm{d}_{6}$ on a Varian Mercury Jeol-(400 MHz) NMR spectrometer. ${ }^{1} \mathrm{H}-\mathrm{NMR}$ (400 MHz) and ${ }^{13} \mathrm{C}-\mathrm{NMR}$ were run in $\left(\mathrm{DMSO}-\mathrm{d}_{6}\right)$. Chemical shifts were related to that of the residual solvent peak. Mass spectra were recorded on a Shimadzu GCMS-QP 1000 EX mass spectrometer at $70 \mathrm{eV}$. Elemental analyses were carried out at the Microanalytical Center of King Saud University, Riyadh, Saudi Arabia.

\subsection{General Procedure for the Reaction of Bis-2-Bromoethanone Derivative $\mathbf{1}$ with Urea, Thiourea and Guanidine: Preparation of Compounds $\mathbf{2 a - c}$}

Compound $1(0.410 \mathrm{~g}, 1 \mathrm{mmol})$ was treated with urea, thiourea or guanidine $(2 \mathrm{mmol})$ in dry ethanol $(20 \mathrm{~mL}, 99.9 \%)$ under reflux for $4-6 \mathrm{~h}$. After addition of TEA $(0.5 \mathrm{~mL})$ the corresponding derivatives 2a-c were formed as solids that were filtered off, washed with ethanol, dried and recrystallized (DMF/EtOH) to afford the desired product in pure form.

4,4'-(3,4-Dimethylthieno[2,3-b] thiophen-2,5-diyl)bis(oxazole-2-amine) (2a). Dark yellow crystals; yield 77\%; mp > $320{ }^{\circ} \mathrm{C}$; IR $(\mathrm{KBr}) v_{\max } 3417,3391\left(\mathrm{NH}_{2}\right) \mathrm{cm}^{-1} ;{ }^{1} \mathrm{H}-\mathrm{NMR}: \delta 2.23\left(\mathrm{~s}, 6 \mathrm{H}, \mathrm{CH}_{3}\right), 7.19$ (s, 2H, oxazole), 6.65 (s, 4H, $\mathrm{NH}_{2}$ aromatic); ${ }^{13} \mathrm{C}-\mathrm{NMR}: \delta 14.8\left(2 \mathrm{CH}_{3}\right.$, aliphatic), 128.8, 134.3, 148.1, 148.8 (thienothiophene ArC's), 136.1, 140.0, 159.3 (ArC's); MS m/z (\%): $332\left(\mathrm{M}^{+}, 6\right), 331$ (51), 317 (100), 165 (48), 76 (98). Anal. for $\mathrm{C}_{14} \mathrm{H}_{12} \mathrm{~N}_{4} \mathrm{O}_{2} \mathrm{~S}_{2}$ (332.40) calcd. C, 50.59; H, 3.64; N, 16.86; S, 19.29. Found: C, 50.48; H, 3.62; N, 16.90; S, 19.20.

4,4'-(3,4-Dimethylthieno[2,3-b]thiophen-2,5-diyl)bis(thiazol-2-amine) (2b). Bright brown crystals; yield $89 \%$; mp. $295{ }^{\circ} \mathrm{C}$; IR $(\mathrm{KBr}) v_{\max } 3420,3391\left(\mathrm{NH}_{2}\right) \mathrm{cm}^{-1}$; ${ }^{1} \mathrm{H}-\mathrm{NMR}$ : $\delta 2.37\left(\mathrm{~s}, 6 \mathrm{H}, \mathrm{CH}_{3}\right), 8.23$ (s, 2H, thiazole), 5.88 (s, $4 \mathrm{H}, \mathrm{NH}_{2}$ aromatic); ${ }^{13} \mathrm{C}-\mathrm{NMR}: \delta 15.2\left(2 \mathrm{CH}_{3}\right.$, aliphatic), 129.8, 133.8, 147.2, 148.1 (thienothiophene ArC's), 135.6, 140.2, 167.3 (ArC's); MS m/z (\%): 365 (M + 1,15), 364 (M, 39), 331 (51), 207 (48), 79 (98). Anal. for $\mathrm{C}_{14} \mathrm{H}_{12} \mathrm{~N}_{4} \mathrm{~S}_{4}$ (364.53) calcd. C, 46.13; H, 3.32; N, 15.37; S, 35.18. Found: C, 46.10; H, 3.34; N, 15.28; S, 35.12.

4,4'-(3,4-Dimethylthieno[2,3-b]thiophen-2,5-diyl)bis(1H-imidazol-2-amine) (2c). Light brown crystals; yield 95\%; mp. $288^{\circ} \mathrm{C}$; IR (KBr) $v_{\max } 3422,3385\left(\mathrm{NH}_{2}\right), 3220(\mathrm{NH}) \mathrm{cm}^{-1}$; ${ }^{1} \mathrm{H}-\mathrm{NMR}: \delta 2.33(\mathrm{~s}, 6 \mathrm{H}$, $\mathrm{CH} 3$ ), 7.68 (s, 2H, imidazole), 6.51 (s, 4H, $\mathrm{NH}_{2}$ aromatic), 12.28 (s, 2H,NH imidazole). ${ }^{13} \mathrm{C}-\mathrm{NMR}$ : $\delta 14.8\left(2 \mathrm{CH}_{3}\right.$, aliphatic), 130.5, 134.3, 148.3, 148.6 (thienothiophene ArC's), 136.3, 141.2, 162.1 (ArC's); MS m/z (\%): 331 (M + 1, 28), 330 (M, 100), 298 (21), 168 (43), 98 (63), 79 (38). Anal. for $\mathrm{C}_{14} \mathrm{H}_{14} \mathrm{~N}_{6} \mathrm{~S}_{2}$ (330.43) calcd. C, 50.89; H, 4.27; N, 25.43; S, 19.41. Found: C, 50.78; H, 4.25; N, 25.38; S, 19.36 . 


\subsection{General Procedure for the Reaction of Bis-2-Bromoethanone Derivative $\mathbf{1}$ wih Aniline and} 2-Aminopyrimidine

Treatment of compound $1(0.410 \mathrm{~g}, 1 \mathrm{mmol})$ with aniline or 2-aminopyrimidine (2 mmol) in dry ethanol (20 mL 99.9\%) at reflux for 5-8 h afforded the corresponding derivatives 4 and 5, respectively. The solid products formed were filtered off, washed with ethanol, dried and recrystallized (DMF/EtOH).

2,2'-(3,4-Dimethylthieno[2,3-b]thiophen-2,5-diyl)bis((3a)H-indole)) (4). Yellow crystals; yield 95\%; $\mathrm{mp}>320{ }^{\circ} \mathrm{C}$; IR $(\mathrm{KBr}) v_{\max } 3390(\mathrm{NH}), 1690(\mathrm{C}=\mathrm{O}) \mathrm{cm}^{-1} ;{ }^{1} \mathrm{H}-\mathrm{NMR}: \delta 2.83\left(\mathrm{~s}, 6 \mathrm{H}, \mathrm{CH}_{3}\right), 7.77-7.83$ (s, 10H, ArH's); $6.33(2 \mathrm{H}, \mathrm{NH}) ;{ }^{13} \mathrm{C}-\mathrm{NMR}: \delta 15.1\left(2 \mathrm{CH}_{3}\right.$, aliphatic), 131.3, 132.3, 144.6, 148.3 (thieno-thiophene ArC's), 34.9, 105.3, 121.2, 122.2, 124.6, 127.2, 166.4 (ArC's); MS m/z (\%): 399 $(\mathrm{M}+1,41), 398(\mathrm{M}, 89), 397$ (84), 383 (24), 165 (54). Anal. for $\mathrm{C}_{24} \mathrm{H}_{18} \mathrm{~N}_{2} \mathrm{~S}_{2}$ (398.54) calcd. C, 72.33; H, 4.55; N, 7.03; S, 16.09. Found: C, 72.22; H, 4.49; N, 7.13; S, 16.03.

2,2'-(3,4-Dimethylthieno[2,3-b]thiophen-2,5-diyl)bis(imidazo[1,2-a]pyrimidine) (5). Brown crystals; yield $78 \%$; mp $>320{ }^{\circ} \mathrm{C}$; IR $(\mathrm{KBr}) v_{\max } 1600(\mathrm{C}=\mathrm{N}) \mathrm{cm}^{-1}$; ${ }^{1} \mathrm{H}-\mathrm{NMR}: \delta 2.97\left(\mathrm{~s}, 6 \mathrm{H}, \mathrm{CH}_{3}\right), 7.78(\mathrm{~s}, 2 \mathrm{H}$, $\mathrm{CH}$, imidazole), 7.43, 7.45, 7.80 (s, 6H, CH, pyrimidine); ${ }^{13} \mathrm{C}-\mathrm{NMR}: \delta 14.3\left(2 \mathrm{CH}_{3}\right.$, aliphatic), 130.2, 133.4, 145.7, 148.4 (thienothiophene ArC's), 103.9, 111.4, 122.2, 127.2, 159.1, 163.2 (ArC's); MS m/z (\%): 403 (M + 1,46), 402 (M, 100), 387 (29), 284 (12). Anal. for $\mathrm{C}_{24} \mathrm{H}_{18} \mathrm{~N}_{2} \mathrm{~S}_{2}$ (402.50) calcd. C, 59.68; H, 3.5; N, 20.88; S, 15.93. Found: C, 59.56; H, 3.48; N, 20.78; S, 15.99.

5,5'-(3,4-Dimethylthieno[2,3-b] thiophen-2,5-diyl)bis(1H-imidazo[1,2-b][1,2,4]triazole (6). Compound $1(0.410 \mathrm{~g}, 1 \mathrm{mmol})$, was added to 4-amino-1,2,4-triazole $(0.168 \mathrm{~g}, 2 \mathrm{mmol})$ in dry ethanol $(20 \mathrm{~mL}$, 99.9\%) at reflux for $4 \mathrm{~h}$. After adding TEA $(0.5 \mathrm{~mL})$, two minutes of heating followed. The solid product formed was filtered off, washed with ethanol, dried and recrystallized (DMF/EtOH). Red crystals; yield 81\%; mp. 228-230 ${ }^{\circ} \mathrm{C}$; IR (KBr) $v_{\max } 3385(\mathrm{NH}) 1560(\mathrm{C}=\mathrm{N}) \mathrm{cm}^{-1}$; ${ }^{1} \mathrm{H}-\mathrm{NMR}$ : $\delta 2.22$ $\left(\mathrm{s}, 6 \mathrm{H}, 2 \mathrm{CH}_{3}\right), 8.52(\mathrm{~s}, 2 \mathrm{H}, \mathrm{CH}$, imidazole) $9.8(\mathrm{~s}, 2 \mathrm{H}, \mathrm{CH}$, triazole $), 12.4(2 \mathrm{H}, \mathrm{NH}$, triazole); ${ }^{13} \mathrm{C}-\mathrm{NMR}$ : $\delta 15.4$ (2 $\mathrm{CH}_{3}$, aliphatic), 137.8, 140.9, 144.4, 148.1 (thienothiophene ArC's), 120.1, 124.1, 156.5, 163.0 (ArC's) MS m/z (\%): 381 (M + 1, 13), 380 (M, 100), 378 (22), 365 (36), 98 (14). Anal. for $\mathrm{C}_{16} \mathrm{H}_{20} \mathrm{~N}_{8} \mathrm{~S}_{2}(380.06)$ calcd. C, 50.51; H, 3.18; N, 29.45; S, 16.86. Found: C, 50.46; H, 3.17; N, 29.22; S, 16.77.

2,2'-(3,4-Dimethylthieno[2,3-b]thiophen-2,5-diyl)bis(9H-benzo[d]imidazo[1,2-a]imidazole) (7). Compound $1(0.410 \mathrm{~g}, 1 \mathrm{mmol})$, was added to 2-aminobenzimidazole $(0.266 \mathrm{~g}, 2 \mathrm{mmol})$ in dry ethanol $(20 \mathrm{~mL}$, $99.9 \%)$ at reflux for $6 \mathrm{~h}$. After adding TEA $(0.5 \mathrm{~mL})$, two minutes of heating followed. The solid product so formed was filtered off, washed with ethanol, dried and recrystallized from (DMF/EtOH). Yellow crystals; yield 78\%; mp > $320{ }^{\circ} \mathrm{C}$; IR $(\mathrm{KBr}) v_{\max } 3414(\mathrm{NH}), 1544(-\mathrm{C}=\mathrm{N}) \mathrm{cm}^{-1}$; ${ }^{1} \mathrm{H}-\mathrm{NMR}$ : $\delta 2.30\left(\mathrm{~s}, 6 \mathrm{H}, 2 \mathrm{CH}_{3}\right), 8.86(2 \mathrm{H}$, imidazole $\mathrm{C}-\mathrm{H}), 12.8(2 \mathrm{H}, \mathrm{NH}$, imidazole $), 7.31,7.33,7.35(4 \mathrm{H}, \mathrm{CH}$, benzimidazole); ${ }^{13} \mathrm{C}-\mathrm{NMR}$ : $\delta 15.88\left(2 \mathrm{CH}_{3}\right.$, aliphatic), 137.8, 140.9, 144.4, 148.1 (thienothiophene ArC's) 107.1, 112.5, 124.1, 124.5, 124.9, 125.0, 157.2 (ArC's); MS m/z (\%): 479 (M + 1, 35), 478 (M, 10), 476 (54), 318 (21), 96 (86). Anal. for $\mathrm{C}_{26} \mathrm{H}_{18} \mathrm{~N}_{6} \mathrm{~S}_{2}$ (478.59) calcd. C, 65.25; H, 3.79; N, 17.56; S, 13.40. Found: C, 65.22; H, 3.67; N, 17.62; S, 13.33 . 


\section{Conclusions}

Syntheses and identification of some bis-heterocycles 2a-c and 4-7 containing thieno[2,3-b]thiophene moieties via the versatile, hitherto unreported reagent 2-bromo-1-[5-(2-bromoacetyl)-3,4-dimethylthieno[2,3-b]thiophen-2-yl]-ethanone (1) were reported.

\section{Acknowledgements}

The authors extend their appreciation to the Deanship of Scientific Research at King Saud University for funding the work through the research group project No. RGP-VPP-007.

\section{References}

1. Mabkhot, Y.N. Synthesis and chemical characterisation of new bis-thieno[2,3-b]thiophene derivatives. Molecules 2010, 15, 3329-3337.

2. Mabkhot, Y.N.; Kheder, N.A.; Al-Majid, A.M. Facile and convenient synthesis of new thieno[2,3-b]-thiophene derivatives. Molecules 2010, 15, 9418-9426.

3. Mabkhot, Y.N. Synthesis and analysis of some bis-heterocyclic compounds containing sulphur. Molecules 2009, 14, 1904-1914.

4. Jarak, I.; Kralj, M.; Piantanida, I.; Suman, L.; Zinic, M.; Pavelic, K.; Karminski-Zamola, G. Novel cyano- and amidino-substituted derivatives of thieno[2,3-b]- and thien-o[3,2-b]thiophene2-carboxanilides and thieno[30,20:4,5]thieno- and thieno[20,30:4,5]thieno[2,3-c]quinolones: Synthesis, photochemical synthesis, DNA binding, and antitumor evaluation. Bioorg. Med. Chem. 2006, 14, 2859-2868.

5. Peters, D.; Hornfeldt, A.B.; Gronowitz, S. Synthesis of various 5-substituted uracils. J. Heterocycl. Chem. 1990, 27, 2165-2173.

6. Kukolja, S.; Draheim, S.E.; Graves, B.J.; Hunden, D.C.; Pfeil, J.L.; Cooper, R.D.G.; Ott, J.L.; Couter, F.T. Orally absorbable cephalosporin antibiotics. 2. Structure-activity studies of bicyclic glycine derivatives of 7-aminodeacetoxycephalosporanic acid. J. Med. Chem. 1985, 28, 1896-1903.

7. Prugh, J.D.; Hartman, G.D.; Mallorga, P.J.; McKeever, B.M.; Michelson, S.R.; Murcko, M.A.; Schwam, H.; Smith, R.L.; Sondey, J.M.; Springer, J.P.; et al. New isomeric classes of topically active ocular hypotensive carbonic anhydrase inhibitors: 5-Substituted thieno[2,3-b]thiophene-2-sulfonamides and 5-substituted thieno[3,2-b]thiophene-2-sulfonamides. J. Med. Chem. 1991, 34, 1805-1818.

8. Hartman, G.D.; Ansdale, P.A. Substituted thieno[2,3-b]thiophene-2-sulfonamides as antiglaucoma agents. U.S. Patent 4,806,562, 1989.

9. Litvinov, V.P. The latest achievements in thienothiophene chemistry. Russ. Chem. Rev. 2005, 74, 217-248.

10. Gather, M.C.; Heeny, M.; Zhang, W.; Whitehead, K.S.; Bradley, D.D.; McCulloch, I.; Campbell, A.J. An alignable fluorene thienothiophene copolymer with deep-blue electrolumenescent emission at $410 \mathrm{~nm}$. Chem. Commun. (Camb.) 2008, 7, 1079-1081.

11. He, M.; Li, J.; Sorensen, M.L.; Zhang, F.; Hancock, R.R.; Fong, H.H.; Pozdin, V.A.; Smilgies, D.; Malliaras, G.G. Alkylsubstituted thienothiophene semiconducting materials: Structure property relationships. J. Am. Chem. Soc. 2009, 131, 11930-11938. 
12. Heeney, M.; Bailey, C.; Genevicius, K.; Shkunov, M.; Sparrowe, D.; Tierney, S.; Mculloch, I. Stable polythiophene semiconductors incorporating thieno[2,3-b]thiophene. J. Am. Chem. Soc. 2005, 127, 1078-1079.

13. Mashraqui, S.H.; Sangvikar, Y.S.; Meetsma, A. Synthesis and structures of thieno[2,3-b]thiophene incorporated [3.3]dithiacyclophanes. Enhanced first hyperpolarizability in an unsymmetrically polarized cyclophane. Tetrahedron Lett. 2006, 47, 5599-5602.

14. Mashraqui, S.H.; Sangvikar, Y.; Ashraf, M.; Kumar, S.; Daub, E. Dipyridyl/pyridinium thieno[2,3-b]thiophenes as new atropisomeric systems. Synthesis, conformat-ional analysis and energy minimization. Tetrahedron 2005, 61, 3507-3513.

15. Leriche, P.R.J.; Turbiez, M.M.; Monroche, V.; Allain, M.; Sauvage, F.X.; Roncali, J.; Frere, P.; Skabara, P.J. Linearly extended tetrathiafulvalene analogues with fused thiophene units as $\pi$-conjugated spacers. J. Mater. Chem. 2003, 13, 1324-1327.

16. Lee, B.; Seshadri, V.; Palko, H.; Sotzing, G.A. Ring-sulfonated poly(thienothiophene). J. Adv. Mater. 2005, 17, 1792-1795.

17. Lim, E.; Jung, B.J.; Lee, J.; Shim, H.K.; Lee, J.I.; Yang, Y.S.; Do, L.M. Thin-film morphologies and solution-processable field-effect transistor behavior of a fluorine-thieno[3,2-b]thiophene-based conjugated copolymer. Macromolecules 2005, 38, 4531-4535.

18. Kim, H.S.; Kim, Y.H.; Kim, T.H.; Noh, Y.Y.; Pyo, S.; Yi, M.H.; Kim, D.Y.; Kwon, S.K. Synthesis and studies on 2-hexylthieno[3,2-b]thiophene end-capped oligomers for OTFTs. Chem. Mater. 2007, 19, 3561-3567.

19. Shefer, N.; Rozen, S. The oxygenation of thieno[2,3-b]thiophenes. J. Org. Chem. 2010, 75, 4623-4625.

20. Hamdya, N.A.; Gamal-Eldeen, A.M.; Abdel-Aziz, H.A.; Fakhr, I.M.I. Modulation of carcinogen metabolizing enzymes by new fused heterocycles pendant to 5,6,7,8-pendant to 5,6,7,8-tetrahydronaphthalene derivatives. Eur. J. Med. Chem. 2010, 45, 463-470.

Sample Availability: Samples of compounds 1-7 are available from the authors.

(C) 2011 by the authors; licensee MDPI, Basel, Switzerland. This article is an open access article distributed under the terms and conditions of the Creative Commons Attribution license (http://creativecommons.org/licenses/by/3.0/). 